\title{
Do successful tuberculosis vaccines need to be immunoregulatory rather than merely Th1-boosting?
}

Graham A. W. Rook, Keertan Dheda, Alimuddin Zumla

Centre for Infectious Disease and International Health, University College London,

Windeyer Institute of Medical Sciences

London WIT 4JF, UK

Correspondence:

\author{
Professor Graham A Rook \\ Centre for Infectious Disease and International Health, \\ University College London, \\ Windeyer Institute of Medical Sciences \\ London WIT 4JF, UK \\ Phone + +442076799489 \\ FAX $\quad+442076368175$ \\ Email_g.rook@ucl.ac.uk
}




\begin{abstract}
Tuberculosis vaccine candidates are entering clinical studies in areas where BCG fails. This is a high risk strategy. We suggest that geographical variation in the efficacy of BCG is related to the presence in developing countries of a cross-reactive background Th2-like response, probably attributable to exposure of mother and infant to helminths and environmental mycobacteria. Such Th2-like activity can stop M. tuberculosis from being pushed into a latent state by the Th1 response, impair bactericidal functions and cause toxicity of TNF- $\alpha$ and pulmonary fibrosis. A successful vaccine, rather than driving a Th1 response, might need to suppress this pre-existing subversive Th2-like component.
\end{abstract}

\title{
Keywords
}

Vaccine, IL-4, IL-482,

\section{Running headline}

Novel vaccine strategy 


\section{Introduction}

\subsection{Immunity to $T B$; the paradox}

Immunity to $M$. tuberculosis requires a Th1 response. Genetic defects in IL-12 or IFN- $\gamma$ pathways lead to susceptibility [1]. However M. tuberculosis rapidly evokes a potent Th1 response, even in those animals or humans who then go on to get the disease. For instance release of IFN- $\gamma$ in response to ESAT-6 is often used as a test for exposure and becomes positive very rapidly [2]. So why do certain people get tuberculosis? A fashionable hypothesis in 2004 would be that the patient has excessive immunoregulatory activity that is suppressing the protective response. Interestingly some patients have very high levels of IL-10 and appear essentially anergic. This does suggest excessive activity of some kind of regulatory T cell. However most patients, far from being anergic, have the necrotising response originally defined by Robert Koch in guinea pigs and subsequently observed in man [3]. This suggests that the relative activity of the regulatory pathways is variable in $\mathrm{TB}$, but whether this activity is high or low, the patient still has the disease. The activity of regulatory $\mathrm{T}$ cells might not therefore be the crucial factor. It seems increasingly likely that $M$. tuberculosis is pathogenic not because it fails to evoke a Th1 response, or causes downregulation of that response, but rather because it evokes a "deliberately" corrupted Th1 response that cannot control the organism and leads instead to immunopathology.

\subsection{Pathogenicity; failure to evoke Th1, or deliberate sabotage?}

It has been apparent since the time of Robert Koch that the response elicited by M. tuberculosis can cause tissue necrosis without killing the bacteria. Similarly the failure of the host immune system to eliminate residual organisms, even after the vast majority have been killed by chemotherapy is a constant paradox and problem, leading to the requirement for 6 months of therapy. The fact that the Koch phenomenon is not protective, and can lead to susceptibility that is greater than that of un-immunised controls, was conclusively demonstrated by many workers in the early decades of the 20th century [4]. More recently studies of pulmonary infection in the Balb/c mouse have led to the observation that after an initial phase of relatively pure Th1 response, which temporarily stops replication of the organisms, the disease progresses again when IL-4 starts to be expressed [5]. Moreover TNF- $\alpha$ became toxic to the animals at the same time. Several other experimental models of infection have also found that toxicity of TNF- $\alpha$ can be associated with the presence of IL-4 in a dominantly Th1-mediated site of inflammation. We have reviewed these data, and the likely mechanisms elsewhere [6]. Subsequent reports noted 
that the presence of IL-4 is not merely a late consequence of progressive infection. In fact if a Th2 response to $M$. tuberculosis, even to a single epitope expressed within the organism, is evoked before challenge with virulent organisms, the subsequent disease is enhanced, particularly immunopathology, weight loss and fibrosis [7]. Since these are important but neglected characteristics of the human disease, we investigated IL-4 KO Balb/c mice, and confirmed that in the absence of functional IL-4 genes, the infection was somewhat attenuated, but more importantly, the toxicity of TNF- $\alpha$ eliminated and fibrosis diminished [8]. The latter is interesting because there is a view that Th2 cytokines are needed for human pulmonary fibrosis, which is striking in TB, whereas IFN- $\gamma$ downregulates fibrosis [9]. These effects will not be apparent in mouse strains where IL-4 is not induced during progressive disease, and most are probably not mediated via STAT-6 [10]. The "non-Th2" effects of IL-4 are signalled via IRS-1 and IRS-2.

\section{The situation in man}

\subsection{IL-4 in human tuberculosis}

Are these findings in Balb/c mice relevant to man, or is human tuberculosis is more like that occurring in some other mouse strain? Is IL-4 a feature of human tuberculosis? We have summarised the mass of data elsewhere [6]. Briefly, TB patients have IL-4-dependent IgE and IgG4 antibody, T cells in the blood that secrete IL-4 in response to Mycobacterium tuberculosis antigens, multiple serological markers of IL-4 activity (such as soluble CD30), both CD4 and CD8 cells that secrete IL-4 in vitro as detected by flow cytometry, IL-4 in lesions by in situ hybridisation, increased IL-4 mRNA by qRT-PCR, even using unstimulated fresh peripheral blood mononuclear cells, and in some instances, free IL-4 in serum at levels that can be measured by ELISA. (Regional differences in the quantity of IL-4 present in TB patients are discussed later).

\subsection{IL-4, susceptibility and immunopathology in man}

Why might IL-4 be critically important in human tuberculosis? IL-4 deactivates macrophages, switches off signalling via TLR-2 and potently downregulates iNOS [11], which may play a crucial role in driving M. tuberculosis into latency [12]. A recent study has revealed that apparently healthy health-care workers whose CD8+ T cells made IL-4 in response to $M$. tuberculosis, went on to develop active disease within 2-4 years [13]. So IL-4 might undermine 
resistance in the early stages of disease. However it might also contribute to immunopathology, as in the Balb/c mouse. The presence of IL-4 in peripheral blood cells, whether detected by qRTPCR, or detected by flow cytometry after stimulation with PMA and calcium ionophore, correlates with cavitary disease $[14,15]$. Moreover, using bronchoalveolar lavage samples to look at the cytokine profile of lymphocytes infiltrating the lungs, the \% of IL-4-secreting cells averaged $14 \%$ in samples from cavitary disease, but only $2-3 \%$ in samples for non-cavitary disease [16]. This leads to the possibility that as in the mouse, the presence of IL-4 contributes to immunopathology within the dominantly Th1-mediated lesions. The IL-4 hypothesis therefore explains the well-known switch in the role of TNF- $\alpha$ from protective to toxic as the disease progresses [17], and the presence of fibrosis in dominantly Th1 lesions [8,9].

\subsection{The role of $I L-4 \delta 2$, an antagonist of $I L-4$}

Furthermore there is a splice variant of IL-4, known as IL-4 2 [14]. This is a natural antagonist of IL-4. During treatment the mRNA for IL-4 decreases, whereas the IL-4 $2 \mathrm{mRNA}$ increases (Dheda, K. et al, unpublished results). Still more revealing is the observation that expression of IL-4 2 mRNA is increased in the peripheral blood of healthy donors who have latent TB, as defined by the presence of a positive ELISPOT IFN- $\gamma$ response to ESAT- 6 . This has been shown by different methods in Ethiopia and in The Gambia $[18,19]$. It implies that despite their healthy appearance, people with latent TB are involved in a "battle" between IL-4 and its inhibitor. We have hypothesised that this IL-4/IL-4 2 ratio might be a factor that determines whether latent TB progresses or remains latent [6]. The detrimental effects of IL-4 listed above might be blocked if there is sufficient expression of IL-482, allowing expression of iNOS that the organisms into the latent state.

\section{The hypothesis and its implications}

\subsection{The importance of the mixed Th1/IL4 response to $\mathrm{M}$. tuberculosis}

The overall hypothesis is that despite rapid induction of a dominant Th1 response, $M$.

tuberculosis may evoke a minor IL-4 response that is sufficient in some individuals to undermine the efficacy of Th1-mediated immunity and cause immunopathology [6]. The recent discovery that the virulent Beijing strains cause human monocytes to express IL-4 and IL-13 is compatible with this [20]. However this simple concept has other implications, and can resolve several 
outstanding paradoxes. We suggest that $M$. tuberculosis exploits and enhances a pre-existing Th2 component that exists in citizens of developing countries.

\subsection{Failure of BCG vaccination in developing countries}

It has been known for decades that the efficacy of BCG vaccination varies in different parts of the world. In general it is least effective in developing countries where it is most needed [21]. There has been no universally accepted explanation for this phenomenon. However in these countries there is a background of Th2 response to some components of M. tuberculosis. This was clearly demonstrated in blood samples from Malawians in which PPD induced IL-5 secretion, whereas little IL-5 was seen in samples from the UK run in parallel (Dockrell, H., personal communication). Worse still, BCG vaccination failed to down-regulate the IL-5 response (Dockrell, H., personal communication). This Th2 activity might be attributable to the exposure of mother and child to helminths. BCG induced a Th2-biased response in babies that had been sensitised in utero to antigens of Wuchereria bancrofti or Schistosoma haematobium because their mothers were suffering from these infections [22]. Presumably contact with cross-reactive environmental mycobacteria would do the same thing in these babies. However these organisms also induce a Th1 response in Malawi that is demonstrably protective [23], and the possible interplay between the Th1 and Th2 components is crucial, and is discussed later.

\subsection{Discordant result of attempts to measure IL-4 in tuberculosis cases from developed and developing countries}

The levels of IL-4 detected in tuberculosis patients, or released by their lymphocytes when stimulated with mycobacterial antigen in vitro, differ markedly in different studies. At one extreme, free IL-4 may be detected easily by ELISA in the patients' serum or in supernatants of patients' peripheral blood mononuclear cells cultured with tuberculosis antigen in vitro (see Table). At the other extreme IL-4 is not detected at all, or only using sensitive quantitative RTPCR, or by flow cytometry after stimulating the cells in the presence of PMA, calcium ionophore and an inhibitor of cytokine export. Interestingly the studies that require the very sensitive methods are those based in Europe or the United States. In contrast, the studies where IL-4 was readily detected by ELISA in serum or in cell culture supernatants, tend to have taken place in developing countries. These points are illustrated in Fig 1, and the source of the information is listed in the table. Clearly some of the patients studied in Europe or the USA will 
themselves have come initially from developing countries, but the distinction remains in the examples we have found, despite this potential dilution of the difference between the two environments.

\subsection{High fatality rates early during treatment in patients in developing countries.}

Death rates from tuberculosis during the first 2 months of treatment are said to be unusually high in many developing countries. If this is true (but see below), it is intriguing to speculate that in patients with a significant background of Th2 response to M. tuberculosis, release of antigen during therapy leads to a systemic Koch phenomenon mediated by IL-4 and TNF- $\alpha$ as outlined earlier. For instance a high mortality is claimed in Malawi [24], where a background of Th2 response to M. tuberculosis has been documented. However there is also a high rate of HIV in this country. Other confounding factors that affect death rates include distance from health care centres, and the severity of the disease at the time of diagnosis. Nevertheless death rates from tuberculosis are probably high in some developing countries even when these factors are taken into account $[25,26]$. In an area of rural South Africa, with a TB control programme and low sero-prevalence rates for HIV, the mortality in those diagnosed with tuberculosis was $13 \%$ [25]. Further studies will be needed to test the hypothesis that there are more deaths in developing countries, due to a systemic Koch phenomenon aggravated by IL-4.

\subsection{Discordant doses of M. tuberculosis required to infect mice in developed and developing countries.}

Another striking difference between some developing countries and the USA, is the dose of $M$. tuberculosis required to establish pulmonary infection in mice. Authors in the USA routinely establish infection using aerosols that deliver 100-200 bacteria to the lungs. This may be an underestimate, and many of the bacilli delivered this way will go to the gastrointestinal tract, so there may be complex immunological events that are ignored. Nevertheless this observation is incompatible with the fact that in Mexico City the same strain of M. tuberculosis, in the same inbred mouse strain, will not establish progressive infection at an intratracheal dose of less than $10^{6}$ bacilli. In fact even a dose as high as 4000 organisms leads to latent non-progressive disease in Mexico City, detectable only by in situ PCR [27]. We suggest that contact with environmental saprophytes in animal facilities in developing countries induces a mixed Th1/Th2 response (mostly Th1), such as that seen in the human population of Malawi [23] and (Dockrell, 
H., personal communication). A low dose infection may be dealt with by the dominant Th1 component. But particulate antigens such as Leishmania [28] or mycobacteria [5] tend to evoke Th2 responses at higher doses. So when a high dose infection is given, there is a greater probability that the Th1 response will be undermined by the activation of the pre-existing Th2 component.

We suggest that mycobacteria- naïve citizens of the USA resemble the immunologically naïve mice in a modern animal facility, and 5\% or so will develop infection following exposure to relatively low doses of M. tuberculosis, with little induction of IL-4. In contrast, the citizens of developing countries rich in mycobacteria and helminths have pre-existing Th1 and Th2 responses induced by these organisms. Under these conditions a high dose of M. tuberculosis is required for infection, which only progresses in those people in whom the TB is able to exploit the pre-existing Th2 component, and so induce an IL-4 response that is large enough to undermine the pre-existing Th1. Beijing strains should be particularly good at this [20]. In some individuals, for reasons that we do not yet understand, the effect of the IL-4 will be blocked by a disproportionate rise in IL-482 [18, 19].

\section{Implications for vaccine design}

The evidence reviewed here suggests that the current studies of Th1-inducing vaccine candidates will fail to reveal anything that can improve on BCG. Induction of Th1 responses may not be relevant, since M. tuberculosis rapidly does this anyway. Furthermore, in experimental animals, manipulations that increase the Th1 response still further than is achieved by BCG vaccine alone, do not provide any additional protection [29, 30]. Enough Th1 is enough. Any further protection requires something else. It might be more important to devise vaccines that switch off the "subversive" Th2 component probably always present in developing countries. This is entirely feasible. Ways of switching off pre-existing Th2 responses by inducing appropriate types of regulatory $\mathrm{T}$ cell have been discovered in studies of allergic disorders, where much larger Th2 responses have been targeted [31]. This regulatory approach to vaccine design will be particularly relevant to therapeutic vaccination. Th1-inducing vaccines make ongoing disease worse [32, 33]. In other words they evoke local and distant Koch phenomena. (The findings of Robert Koch in the 1890 's are rediscovered at regular intervals). Interestingly the only vaccine candidates shown to be effective therapeutically in the Balb/c mouse TB model both work at least in part by 
suppressing the IL-4 response [34, 35], which BCG, studied in parallel, failed to do [35]. In conclusion the time may have come for a radical reappraisal of the thinking behind the current wave of "rationally" designed vaccine candidates.

\section{References}

[1] de Jong, R, Altare, F, Haagen, IA et al. Severe mycobacterial and Salmonella infections in interleukin-12 receptor-deficient patients. Science 1998;280:1435-1438.

[2] Pathan, AA, Wilkinson, KA, Wilkinson, RJ et al. High frequencies of circulating IFNgamma-secreting CD8 cytotoxic T cells specific for a novel MHC class I-restricted Mycobacterium tuberculosis epitope in M. tuberculosis-infected subjects without disease. Eur J Immunol 2000;30(9):2713-2721.

[3] Anderson, MC. On Koch's treatment. Lancet 1891;i:651-652.

[4] Wilson, GS, Schwabacher, H \& Maier, I. The effect of the desensitisation of tuberculous guinea-pigs. J. Path. Bact. 1940;50:89-109.

[5] Hernandez-Pando, R, Pavon, L, Arriaga, K, Orozco, H, Madrid-Marina, V \& Rook, GAW. Pathogenesis of tuberculosis in mice exposed to low and high doses of an environmental mycobacterial saprophyte. Infect. Immun. 1997;65:3317-3327.

[6] Rook, GAW, Hernandez Pando, R, Dheda, K \& Seah, GT. IL-4 in tuberculosis: implications for vaccine design. Trends in Immunology 2004;25:483-488.

[7] Wangoo, A, Sparer, T, Brown, IN et al. Contribution of Th1 and Th2 Cells to Protection and Pathology in Experimental Models of Granulomatous Lung Disease. J. Immunol. 2001;166:3432-3439.

[8] Hernandez-Pando, R, Aguilar, D, Garcia Hernandez, ML, Orozco, H \& Rook, GAW. Pulmonary tuberculosis in Balb/c mice with non-functional IL-4 genes; changes in the inflammatory effects of TNF- $\alpha$ in the regulation of fibrosis. Eur. J. Immunol. 2004;34:174-183.

[9] Lee, CG, Homer, RJ, Zhu, Z et al. Interleukin-13 induces tissue fibrosis by selectively stimulating and activating transforming growth factor beta(1). J. Exp. Med. 2001;194(6):809-821.

[10] Jung, YJ, LaCourse, R, Ryan, L \& North, RJ. Evidence inconsistent with a negative influence of $\mathrm{T}$ helper 2 cells on protection afforded by a dominant $\mathrm{T}$ helper 1 response against Mycobacterium tuberculosis lung infection in mice. Infect Immun 
2002;70(11):6436-6443.

[11] Bogdan, C, Vodovotz, Y, Paik, J, Xie, QW \& Nathan, C. Mechanism of suppression of nitric oxide synthase expression by interleukin-4 in primary mouse macrophages. J. Leukoc. Biol. 1994;55(2):227-233.

[12] Voskuil, MI, Schnappinger, D, Visconti, KC et al. Inhibition of respiration by nitric oxide induces a Mycobacterium tuberculosis dormancy program. J Exp Med 2003;198(5):705713.

[13] Ordway, D, Costa, D, Martins, M et al. Increased IL-4 production by CD 8 and $\gamma \delta \mathrm{T}$ cells in health care workers is associated with the subsequent development of active tuberculosis. J Infect Dis 2004;in press.

[14] Seah, GT, Scott, GM \& Rook, GA. Type 2 Cytokine Gene Activation and Its Relationship to Extent of Disease in Patients with Tuberculosis. J. Infect. Dis. 2000;181:385-389.

[15] van Crevel, R, Karyadi, E, Preyers, F et al. Increased production of interleukin 4 by CD4+ and CD8+ T cells from patients with tuberculosis is related to the presence of pulmonary cavities. J Infect Dis 2000;181(3):1194-1197.

[16] Mazzarella, G, Bianco, A, Perna, F et al. T lymphocyte phenotypic profile in lung segments affected by cavitary and non-cavitary tuberculosis. Clin Exp Immunol 2003;132(2):283-288.

[17] Tramontana, JM, Utaipat, U, Molloy, A et al. Thalidomide treatment reduces tumor necrosis factor alpha production and enhances weight gain in patients with pulmonary tuberculosis. Mol. Med. 1995;1(4):384-397.

[18] Demissie, A, Abebe, M, Aseffa, A et al. Healthy individuals that control latent infection with M. tuberculosis express high levels of Th1 cytokines and the IL-4 antagonist IL4delta2. J Immunol 2004;172:6938-6943.

[19] Fletcher, HA, Owiafe, P, Jeffries, D et al. Increased expression of mRNA encoding IL-4 and its splice variant IL-4 2 in cells from contacts of Mycobacterium tuberculosis in the absence of in vitro stimulation. Immunology 2004;112:669-673.

[20] Manca, C, Reed, MB, Freeman, S et al. Differential Monocyte Activation Underlies Strain-Specific Mycobacterium tuberculosis Pathogenesis. Infect Immun 2004;72(9):5511-5514.

[21] Wilson, ME, Fineberg, HV \& Colditz, GA. Geographic latitude and the efficacy of bacillus Calmette-Guerin vaccine. Clin Infect Dis 1995;20(4):982-991. 
[22] Malhotra, I, Mungai, P, Wamachi, A et al. Helminth- and Bacillus Calmette-Guerininduced immunity in children sensitized in utero to filariasis and schistosomiasis. $\mathrm{J}$ Immunol 1999;162(11):6843-6848.

[23] Fine, PE, Floyd, S, Stanford, JL et al. Environmental mycobacteria in northern Malawi: implications for the epidemiology of tuberculosis and leprosy. Epidemiol Infect 2001;126(3):379-387.

[24] Harries, AD, Hargreaves, NJ, Gausi, F, Kwanjana, JH \& Salaniponi, FM. High early death rate in tuberculosis patients in Malawi. Int J Tuberc Lung Dis 2001;5(11):10001005.

[25] Barker, RD \& Millard, FJ. High death rates for tuberculosis patients in rural South Africa. Int J Tuberc Lung Dis 1998;2(12):1049-1050.

[26] Wilkinson, D \& Moore, DA. HIV-related tuberculosis in South Africa--clinical features and outcome. S Afr Med J 1996;86(1):64-67.

[27] Arriaga, AK, Orozco, EH, Aguilar, LD, Rook, GAW \& Hernandez Pando, R. Immunological and pathological comparative analysis between experimental latent tuberculous infection and progressive pulmonary tuberculosis. Clin. Exp. Immunol. 2002;128(2):229-237.

[28] Bretscher, PA. A strategy to improve the efficacy of vaccination against tuberculosis and leprosy. Immunol. Today. 1992;13:342-345.

[29] Leal, IS, Smedegard, B, Andersen, P \& Appelberg, R. Failure to induce enhanced protection against tuberculosis by increasing T-cell-dependent interferon-gamma generation. Immunology 2001;104(2):157-161.

[30] Skinner, MA, Ramsay, AJ, Buchan, GS et al. A DNA prime-live vaccine boost strategy in mice can augment IFN-gamma responses to mycobacterial antigens but does not increase the protective efficacy of two attenuated strains of Mycobacterium bovis against bovine tuberculosis. Immunology 2003;108(4):548-555.

[31] Zuany-Amorim, C, Sawicka, E, Manlius, C et al. Suppression of airway eosinophilia by killed Mycobacterium vaccae-induced allergen-specific regulatory T-cells. Nat Med 2002;8:625-629.

[32] Moreira, AL, Tsenova, L, Aman, MH et al. Mycobacterial antigens exacerbate disease manifestations in Mycobacterium tuberculosis-infected mice. Infect Immun 2002;70(4):2100-2107. 
[33] Turner, J, Rhoades, ER, Keen, M, Belisle, JT, Frank, AA \& Orme, IM. Effective preexposure tuberculosis vaccines fail to protect when they are given in an immunotherapeutic mode. Infect. Immun. 2000;68(3):1706-1709.

[34] Hernandez-Pando, R, Pavon, L, Orozco, EH, Rangel, J \& Rook, GAW. Interactions between hormone-mediated and vaccine-mediated immunotherapy for pulmonary tuberculosis in Balb/c mice. Immunology 2000;100:391-398.

[35] Lowrie, DB, Tascon, RE, Bonato, VL et al. Therapy of tuberculosis in mice by DNA vaccination. Nature 1999;400:269-271. 


\section{Figure legend}

Geographical distribution of tuberculosis patients in whom IL-4 production was massively increased (i.e. detectable by simple ELISA assay or equivalent method) (black triangles) or required RT-PCR or prestimulation and flow cytometry (red circles). The location of the sites, and the references, are listed in the Table. The dashed lines are drawn at approximately $30^{\circ}$ North and South of the equator. 


\section{Table 1}

\begin{tabular}{|l|l|l|}
\hline Abbreviated reference: Developed countries & Country & Assay method \\
\hline * Schauf V et al, 1993, J. Infect. Dis, 168: 1056. & USA & RT-PCR \\
* Barnes PF et al, 1993, Infect Immun, 61:197. & USA & RT-PCR \\
* Verbon A et al, 1999, Clin Exp Immunol, 115:110. & Netherlands & Serum IL-4 not detect by ELISA \\
* van Crevel R et al, 2000, J Infect Dis, 181:1194. & Netherlands & FACS after mitogen stimulation \\
* Seah GT et al, 2000, J Infect Dis, 181:385. & UK & Nested RT-PCR \\
* Surcel HM et al, 1994, Immunology, 81:171. & UK & ELISPOT \\
* Silveira H et al, 1997, Clin Exp Immunol, 110:26. & Portugal & RT-PCR \\
\hline Abbreviated reference: Developing countries & Country & AsSay method \\
\hline * Jimenez-Martinez MC et al, 2004, Immunology, 111:100. & Mexico & FACS after stim with TB antigen \\
* Dlugovitzky D et al, 1999, Scand J Immunol, 49:210. & Argentina & ELISA \\
* Dlugovitzky D et al, 2004, Repir Med, in press. & Argentina & ELISA \\
* Montiel M et al, 2002, Acta Cient Venez, 53:36. & Venezuela & ELISA \\
* Sanchez FO et al, 1994, Infect Immun, 1994, 62:5673. & Columbia & ELISA \\
* Smith SM et al, 2002, Tuberculosis, 82:7. & The Gambia & FACS after stim with $M . t b$ \\
* Bhattacharyya S et al, 1999, Infect Immun, 67:5597. & India & FACS without antigen stim. \\
* Sharma SK et al, 2002, J Clin Immunol, 22:345. & India & FACS without antigen stim. \\
* Wang L et al, 2002, Zhonghua Jie He He Hu Xi Za Zhi, & China & ELISA \\
25:535. & & \\
\hline
\end{tabular}


Fig 1

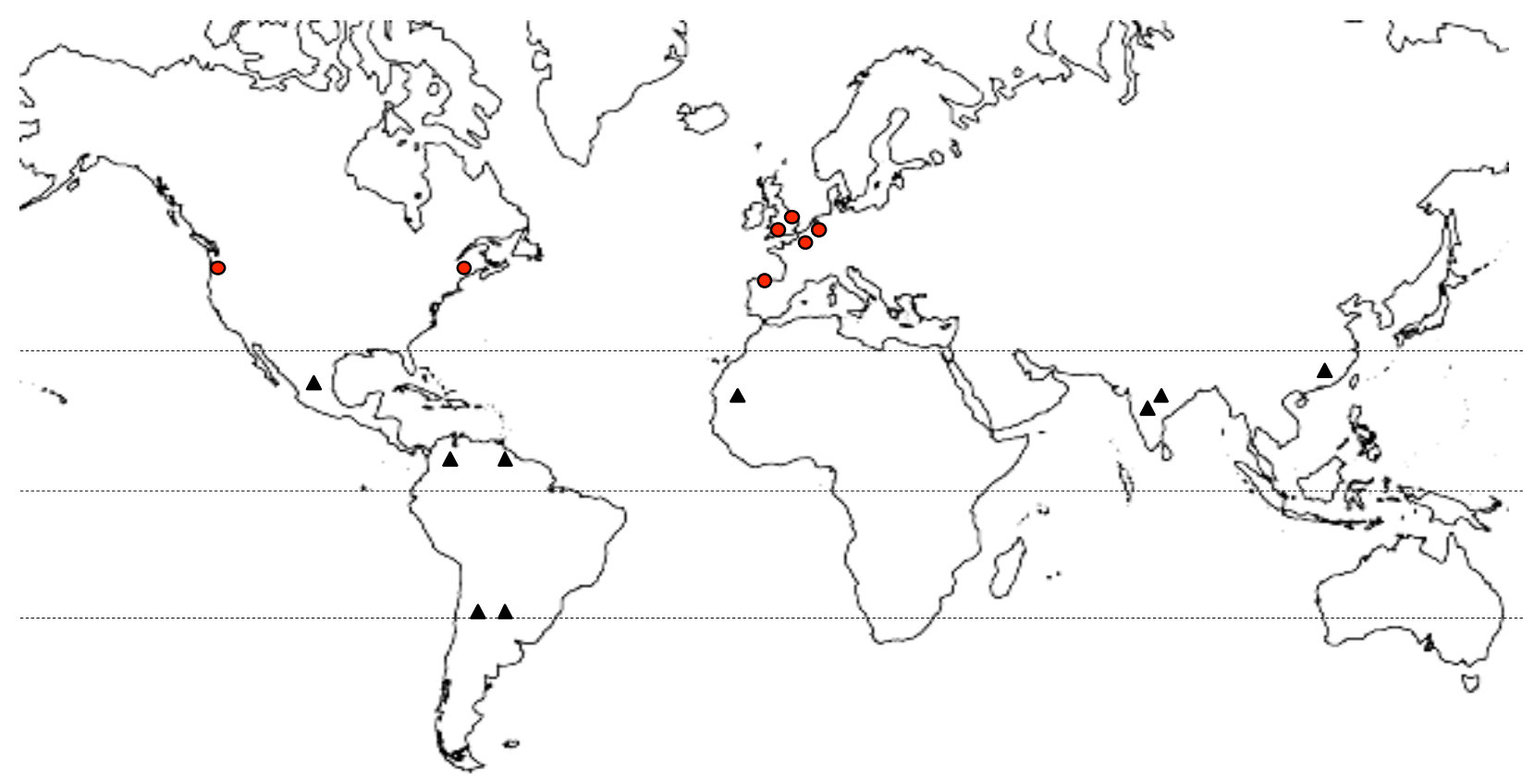

